\title{
Infrared Study of the Stability and Folding Kinetics of a 15-Residue $\beta$ - Hairpin
}

\author{
Yao Xu, Rolando Oyola, and Feng Gai* \\ Department of Chemistry, University of Pennsylvania, Philadelphia, PA 19104
}

Supporting Information

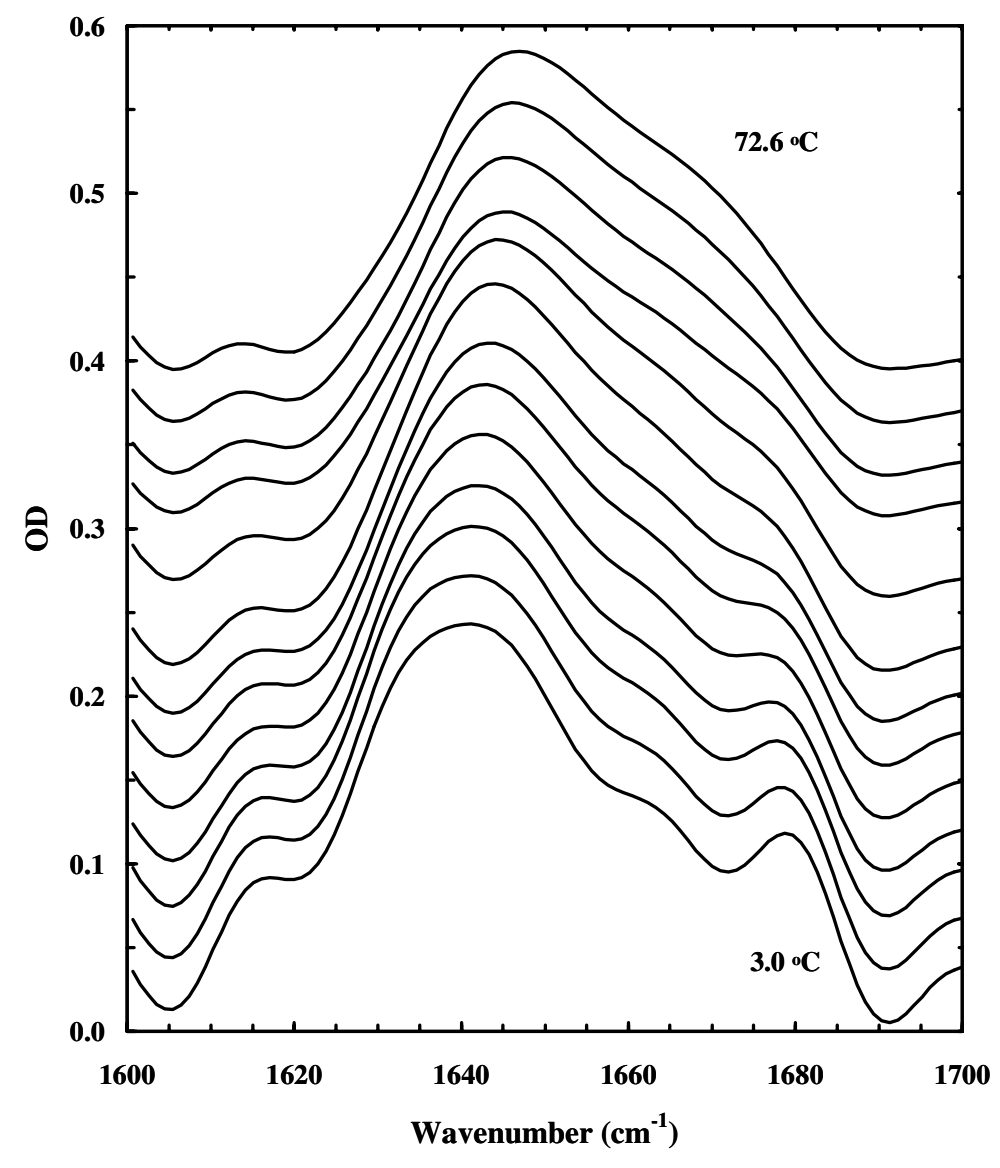

Figure1. Resolution enhanced amide I' spectra of peptide 1 in $\mathrm{D}_{2} \mathrm{O}$ phosphate buffer solution (pH* 7) from 3 to $72.6{ }^{\circ} \mathrm{C}$ in $5.8{ }^{\circ} \mathrm{C}$ steps. Resolution enhancements were archived by Fourier Self-deconvolution with $\mathrm{k}=2$ and bandwidth $=24 \mathrm{~cm}^{-1}$. A band around $1664 \mathrm{~cm}^{-1}$ is evident. 
(a)

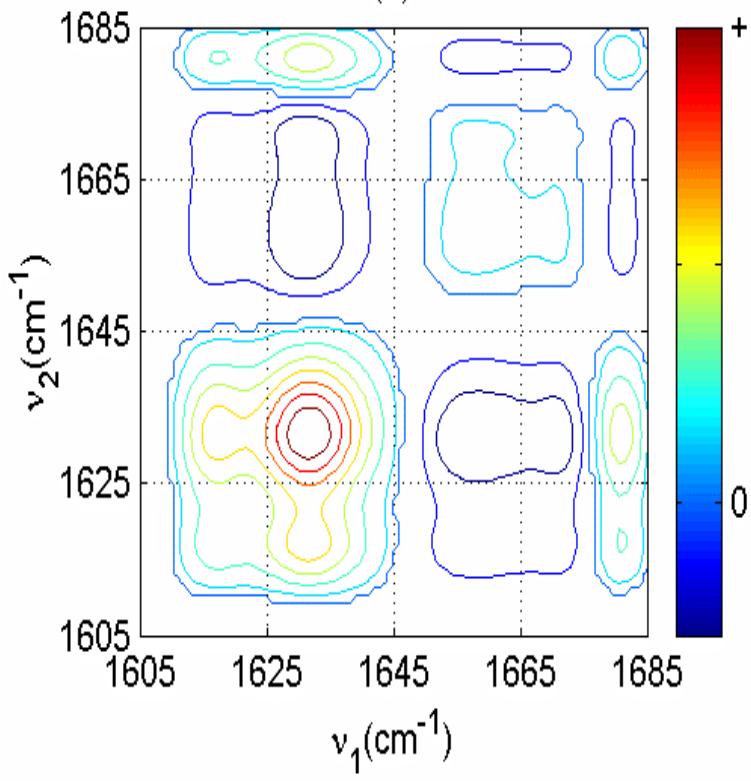

(b)

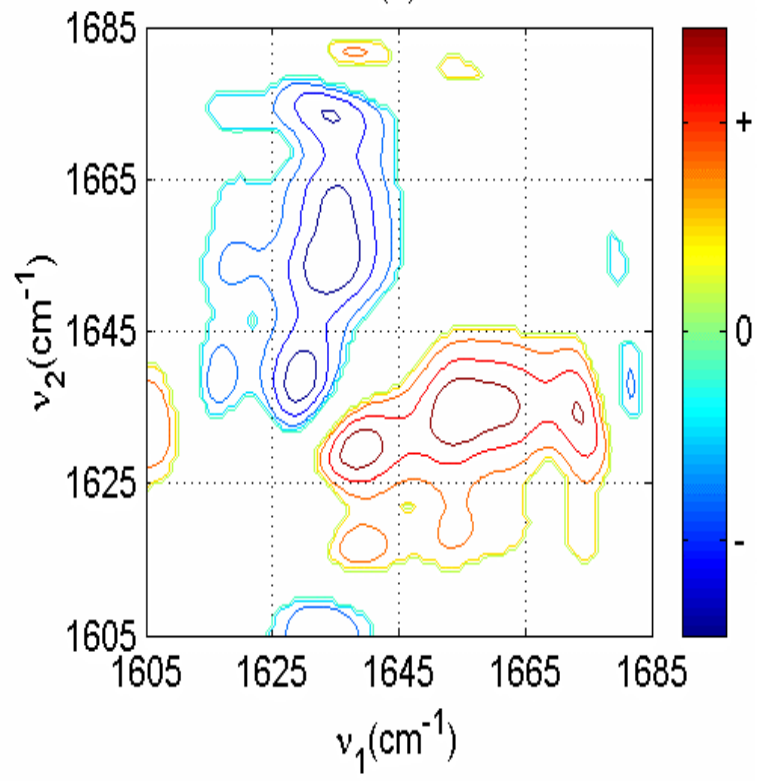

Figure 2. $2 D^{1}$ IR (a) synchronous and (b) asynchronous plots of peptide $\mathbf{1}$ in $\mathrm{D}_{2} \mathrm{O}$ phosphate buffer solution $\left(\mathrm{pH}^{*} 7\right)$. These data indicate that the intensity of a spectral feature around 1664 $\mathrm{cm}^{-1}$ increases with increasing temperature.
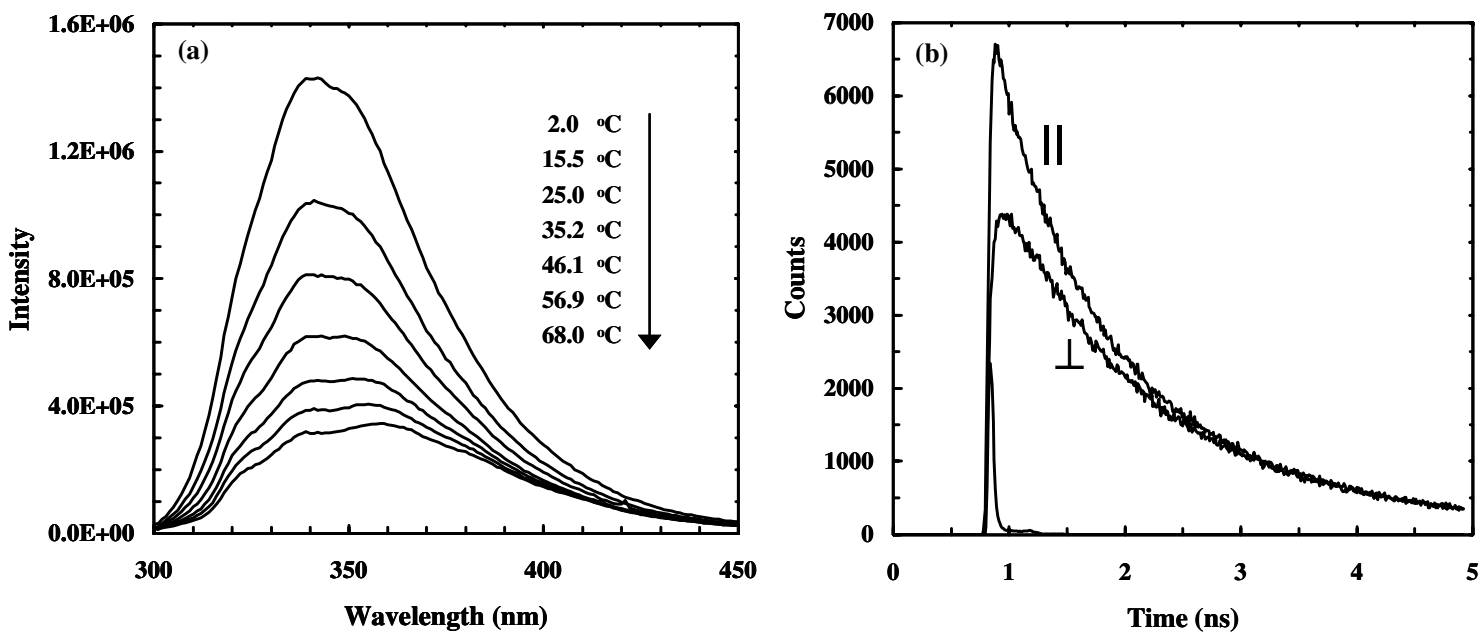

Figure 3. (a) Temperature dependent fluorescence spectra of peptide $\mathbf{1}$ in $\mathrm{D}_{2} \mathrm{O}$ phosphate buffer solution (pH* 7). $\lambda_{\mathrm{ex}}=295 \mathrm{~nm}$. (b) Parallel and perpendicular fluorescence decays of peptide 1 in $\mathrm{D}_{2} \mathrm{O}$ phosphate buffer solution $\left(\mathrm{pH}^{*} 7\right) . \lambda_{\mathrm{ex}}=295 \mathrm{~nm}, \lambda_{\mathrm{em}}=350 \mathrm{~nm}$. Globally fitting these data yields the following anisotropy decay: $r(t)=0.17 \exp (-t / 100 \mathrm{ps})+0.11 \exp (-\mathrm{t} / 1.1 \mathrm{~ns})$. The longer component corresponds to the overall rotation of the peptide, whereas the shorter component is due to the internal motion of Trp side chain. An anisotropy of 0.17 gives rise to a solid angle of 33 degrees. ${ }^{2}$

\footnotetext{
${ }^{1}$ Noda, I. Appl. Spectrosc. 1993, 47, 1329-1336.

${ }^{2}$ Lipari, G.; Azabo, A. J. Am. Chem. Soc. 1982, 104, 4559-4570.
} 


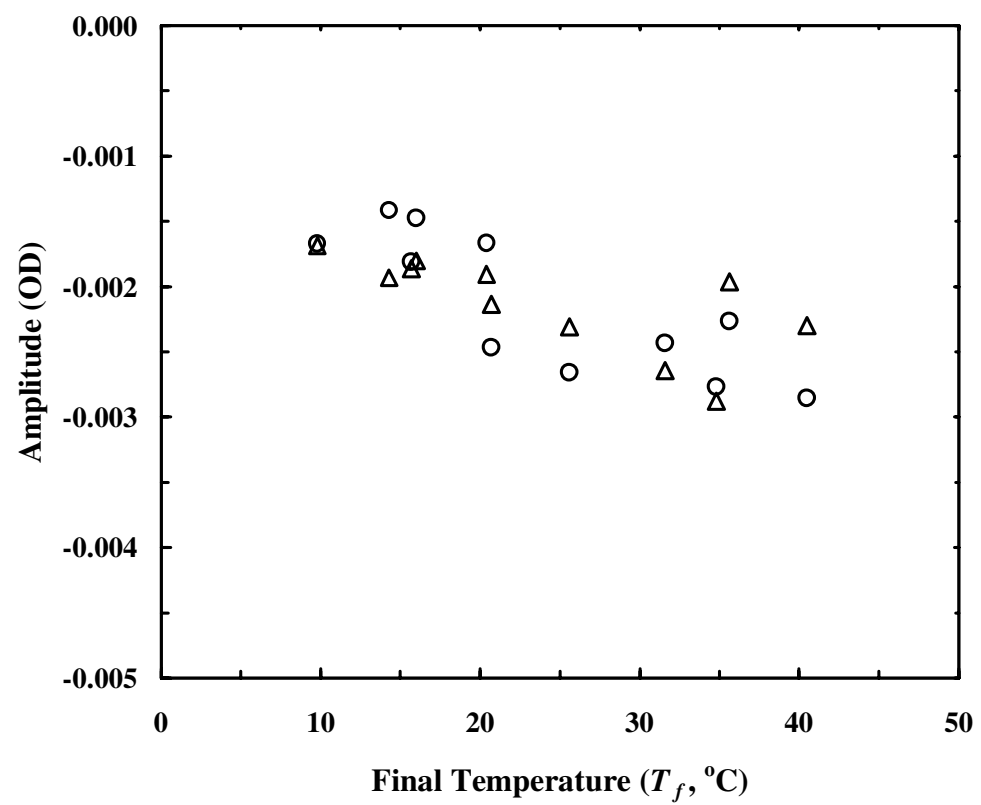

Figure 4. Amplitudes (O) of the slow relaxation kinetics following a $T$-jump between $T_{i}$ and $T_{f}$ and the expected (and scaled) $\beta$-hairpin population changes $(\Delta)$ corresponding to the same $T$ jump calculated from the thermodynamic parameters obtained by the current IR method.
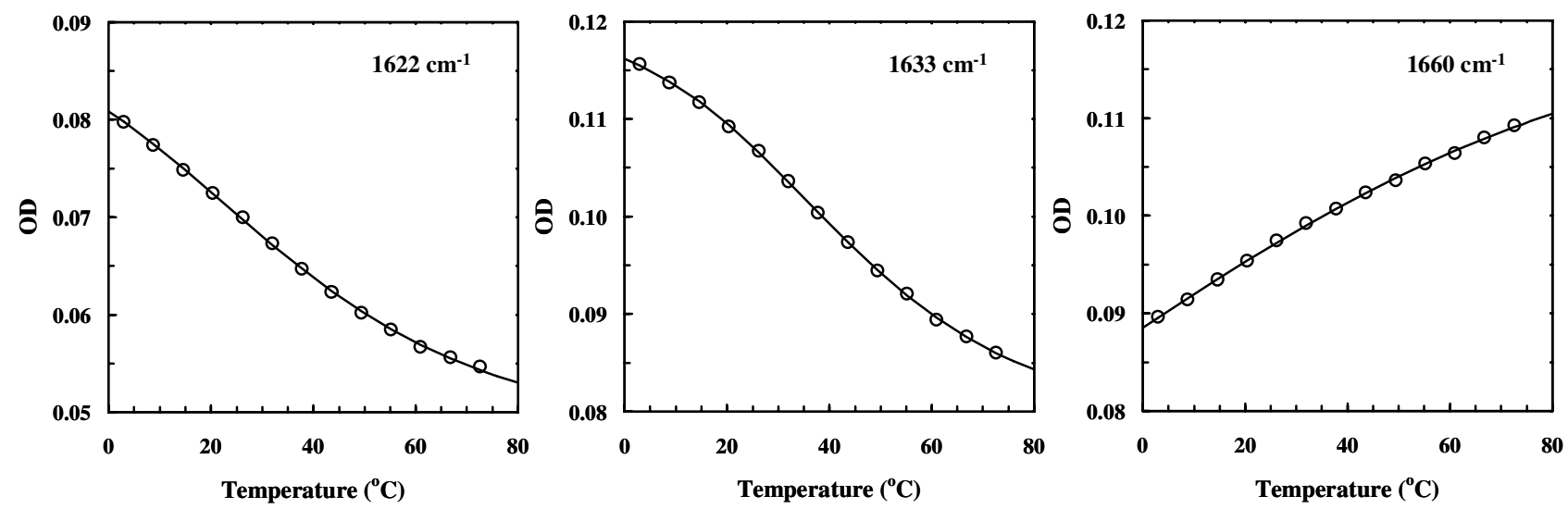

Figure 5. Thermal denaturations probed by optical density changes at individual frequencies, as indicated. The lines are fits to a two-state model with temperature independent baselines. The resulting thermodynamic parameters are: $1622 \mathrm{~cm}^{-1}: T_{m}=30.0^{\circ} \mathrm{C}, \Delta H_{m}=-7.79 \mathrm{kcal} \mathrm{mol}^{-1}, \Delta S_{m}=$ $-25.7 \mathrm{cal} \mathrm{mol}^{-1} \mathrm{~K}^{-1}$, and $\Delta C_{p} \approx 0 ; 1633 \mathrm{~cm}^{-1}: T_{m}=41.5^{\circ} \mathrm{C}, \Delta H_{m}=-10.1 \mathrm{kcal} \mathrm{mol}^{-1}, \Delta S_{m}=-32.1$ cal mol ${ }^{-1} \mathrm{~K}^{-1}$, and $\Delta C_{p} \approx 0 ; 1660 \mathrm{~cm}^{-1}: T_{m}=25.3{ }^{\circ} \mathrm{C}, \Delta H_{m}=-3.94 \mathrm{kcal} \mathrm{mol}^{-1}, \Delta S_{m}=-13.2 \mathrm{cal}$ $\mathrm{mol}^{-1} \mathrm{~K}^{-1}$, and $\Delta C_{p} \approx 0$. When temperature dependent baselines were used, a range of parameters could yield almost equally good fits. Taken together, these results indicate that it is not reliable to obtain thermal unfolding thermodynamics from absorbance changes at a single frequency. 